\title{
Dietary administration of astaxanthin improves feed utilization, growth performance and survival of Asian seabass, Lates calcarifer (Bloch, 1790)
}

\begin{abstract}
This study was undertaken to assess the impacts of dietary astaxanthin supplementation on growth performance, feed utilization, survival, and serum growth hormone $(\mathrm{GH})$ availability of Asian seabass, Lates calcarifer, with special reference to dose-response relationships and variations during different feeding phases (short-term, medium-term and long-term). Fish were fed the following diets in triplicate for 90 days: the control (CD), AX50 (50 mg astaxanthin/kg diet), AX100 (100 mg astaxanthin/ $/ \mathrm{kg}$ diet) and AX150 (150 mg astaxanthin $/ \mathrm{kg}$ diet). The findings revealed that fish exhibited significant linear increments $(\mathrm{p}<.05)$ in specific growth rate (SGR), weight gain, feed utilization efficiency and survival when fed various diets with escalating levels of astaxanthin. Supplementation with dietary astaxanthin significantly augmented ( $\mathrm{p}<$ $.05) \mathrm{GH}$ levels in fish. Significant positive associations ( $p<.05)$ were observed between circulating serum GH levels and SGR of fish from all groups following three consecutive feeding phases, denoting a robust cause-and-effect relationship. Circulating GH concentrations were considered as a sensitive biomarker of growth performance in Asian seabass. This study illustrated that supplemental astaxanthin could be administered in culture protocols to improve the growth rate and commercial hatchery production of Asian seabass, and possibly other teleost species.
\end{abstract}

Keyword: Asian seabass; Astaxanthin; Feed utilization; Growth hormone; Growth performance; Survival 